\title{
How skin and object temperature influence touch sensation
}

\author{
JOSEPH C. STEVENS and JANET E. HOOPER \\ John B. Pierce Foundation Laboratory, New Haven, Connecticut \\ and Yale University, New Haven, Connecticut
}

\begin{abstract}
Twenty subjects made magnitude estimations of the apparent heaviness of metallic stimulators of various masses and temperatures (cold, neutral, and warm). The stimulators were placed on the forearm at normal skin temperature (about $33^{\circ} \mathrm{C}$ ), at elevated skin temperature $\left(38^{\circ} \mathrm{C}\right)$, and at lowered skin temperature $\left(25^{\circ} \mathrm{C}\right)$. Under neutral conditions, we replicated earlier studies: cold objects felt considerably heavier than neutral ones, warm objects somewhat heavier than neutral objects. Warming the skin essentially obliterated the intensifying effect of the warm objects but left unaltered the intensifying effect of the cold objects. Cooling the arm also essentially obliterated the intensifying effect of warm objects. It also diminished, but far from eliminated, the intensifying effect of cold objects. Cold intensification of touch sensation is a large and robust phenomenon and, unlike warm intensification, appears to characterize all body regions.
\end{abstract}

In 1846, E. H. Weber reported that a cold German dollar (Thaler) placed on the forehead felt approximately as heavy as two warm dollars, one on top of the other. Weber conjectured that cooling strengthens and warming lightens pressure sensations. We shall see that his conjecture is half right and half wrong, but it is curious that the sheer magnitude of Weber's observation failed to prompt much further investigation of the effects of temperature on touch sensation (for a brief history, see Stevens \& Green, 1978).

The phenomena took on fresh interest, however, as it became apparent to many students of the dermal nerves that individual dibers often respond to more than one kind of stimulation, that is, show imperfect stimulus selectivity. The outstanding example is that mechanoreceptors commonly respond not only to touch, but also to cooling of the skin. Other types of fibers also show response to multimodal stimulation (for a review of the physiological literature, see Stevens \& Green, 1978). Could it be that imperfect stimulus selectivity account for the Weber phenomenon?

Questions of this sort led to a large set of experiments in this laboratory (Stevens, 1979a, 1982; Stevens \& Green, 1978). Four general principles have emerged: (1) Cold does intensify pressure sensation, and the colder the object, the heavier it feels. This part of Weber's conjecture is beyond doubt true. A strong effect was noted in seven body regions. In the extreme, a cold 10-g weight matched

This research was supported by NIH Grant R01NS15419. Requests for reprints should be addressed to Joseph C. Stevens, John B. Pierce Foundation Laboratory, 290 Congress Avenue, New Haven, Connecticut 06519. a neutral (i.e., at skin temperature) 100 -g weight. (2) Warmth can also intensify pressure sensation, but the effect is considerably smaller and appears to characterize only some body regions. For example, we have demonstrated it clearly in the forearm but not in the forehead. Never have we observed that warmth (above skin temperature) lightened a pressure sensation. This part of Weber's conjecture seems to be wrong. (3) Under comparable conditions of stimulation, there appears to be no "reverse" Weber phenomenom; that is, changing the force of stimulation does not alter the sensation of warmth or cold (Zimmermann \& Stevens, 1982). This finding, coupled with the failure of warmth to intensify pressure in some body regions, suggests that intensification is not the simple addition of two concurrent sensations. Rather, imperfect stimulus selectivity of mechanoreceptors would seem to be a more likely explanation. (4) On this view, we hypothesized that temperature could be used to sharpen the spatial acuity of the skin (Stevens, 1982). This indeed proved true in measurements of two-point and two-edge thresholds on the forearm. Both warm and cold improved acuity by an average of about $40 \%$. That this represents thermal effects on mechanoreceptors seemed likely because pure thermal sensations show virtually no acuity (Cain, 1973; Vendrik \& Eijkman, 1968). Thus, it may be that the function of imperfect stimulus selectivity is basically to sharpen the tactile world and that temperature might be deliberately invoked as a practical means of improving acuity.

Unexplored as yet was the question of how skin temperature and object temperature combine their effects to produce thermal intensification. From casual impressions, we drew the conclusion that warm- 
ing the skin might reduce or eliminate thermal intensification by warmth on the forearm (Stevens \& Green, 1978). The same could be true of cold. The implications are that prolonged inspection of warm or cold objects might reduce the thermal intensification and thermal sharpening of acuity. However, there is also the question of whether warming the skin might promote intensification by cold objects and cooling the skin promote intensification by warm objects. In addition to being of psychophysical and physiological interest, the answers to these questions would be helpful in choosing the optimal kinds of temperature stimulation that might be put to use in tactile communication systems, such as braille or the opticon, in which resolution of the tactile elements is one of the chief limiting factors. That is to say, if the elements of a display are crowded too closely together, they cannot be resolved, and spreading them out too far may prohibit good perceptual integration of the pattern.

\section{METHODS}

Each of 20 subjects (13 female, 7 male) served in two essentially equivalent test sessions on separate days. A session lasted approximately $1 \mathrm{~h}$.

There were three parameters of stimulation: (1) weight of circular aluminum stimulators $(21,45$, and $105 \mathrm{~g}),(2)$ temperature of these stimulators (warm, neutral, cold), and (3) temperature of the subject's forearm (warm, neutral, and cold). The surface area of all stimulators was $12.6 \mathrm{~cm}^{2}$. The stimulators were placed so as to sink, of their own weight, into the skin of the volar forearm for about 2 sec. As in the earlier studies of the Weber phenomenon, the subjects were instructed to make magnitude estimations of the apparent heaviness of the weights with no designated standard and without regard to temperature sensation.

The temperature of the weights was controlled by regulated water baths (at $34^{\circ}$ and $45^{\circ} \mathrm{C}$ ) or ice water $\left(0^{\circ}\right)$. (The actual change in skin temperature is approximately proportional to the temperature of the weights but is less than the difference between the skin and the weight temperatures. For a discussion of the relation between stimulator temperature and skin temperature, see Stevens, $1979 \mathrm{~b}$.) The $34^{\circ} \mathrm{C}$ stimulator felt neutral, the $45^{\circ}$ distinctly warm, the $0^{\circ}$ stimulator distinctly cold, although never noxious.

The temperature of the skin was controlled either by simple exposure to normal ambient air or by inserting the arm through a port in a small air-conditioned box $(65 \mathrm{~cm}$ long $\times 38 \mathrm{~cm} \times 38 \mathrm{~cm})$. Skin temperature was controlled by a combination of convective cooling (cold air vented from an air conditioner) and radiant heating from a heat lamp mounted above the subject's arm. A thermistor taped on the arm drove a proportional controller, which, in turn, regulated the intensity of the heat lamp. In this way, a wide range of skin temperatures could be achieved with good precision (to within about $.25^{\circ} \mathrm{C}$, in the present experiment). A thermocouple near the site of stimulation served to monitor skin temperature on a strip-chart recorder. A second port in the chamber allowed the experimenter to place the weights on the subject's arm. Skin temperatures were $25^{\circ} \mathrm{C}$ and $38^{\circ} \mathrm{C}$ (stabilized for at least $2 \mathrm{~min}$ before testing began), as compared with an average of $33^{\circ} \mathrm{C}$ for the arm in neutral ambient air.

A session began with a comparison of the left and right arms at ordinary ambient temperatures. The reasons for this were: (1) to establish a body of control data obtained under conditions similar to those of earlier experimentation, and (2) to control for the possibility of systematic differences in weight sensitivity between the right and left arms. Previous work (Stevens, 1979a) had suggested a right-arm superiority, but the present study did not confirm it. All three weights were presented at all three temperatures two times to each arm, in irregular order but alternating from one arm to the other (a total of 36 presentations and magnitude estimations). Then the subject inserted one arm in either the cold or the warm chamber, and, after stabilization, the weights were again applied, as in the neutral condition, alternately between the left and right arms (another set of 36 presentations). Then the skin temperature was changed from $28^{\circ} \mathrm{C}$ to $45^{\circ} \mathrm{C}$, or vice versa (order counterbalanced across subjects), and a third set of 36 presentations was made (a total of 108 for a session). The second session was the same except that the arm in the chamber in the first session was now kept in neutral ambient air and vice versa.

\section{RESULTS}

Plots of the data for the arm at neutral temperature (control data) showed no differences when the other arm was cold, warm, or neutral. (Differences could not be ruled out a priori, for cooling or warming one arm could conceivably influence the other by way of consensual alterations in blood flow to the skin.) Therefore, all the control data taken in neutral conditions were averaged (geometric means). These are plotted in log-log coordinates in Figure 1, and fitted by straight lines determined by the method of least squares.

The results are in good agreement with comparable conditions in earlier studies. Each cold weight was judged heavier than its neutral counterpart, at high levels of statistical confidence $\left(p<10^{-5}\right.$ for each of the three pairs, as determined by $t$ tests). Each warm weight was also judged heavier than its neutral

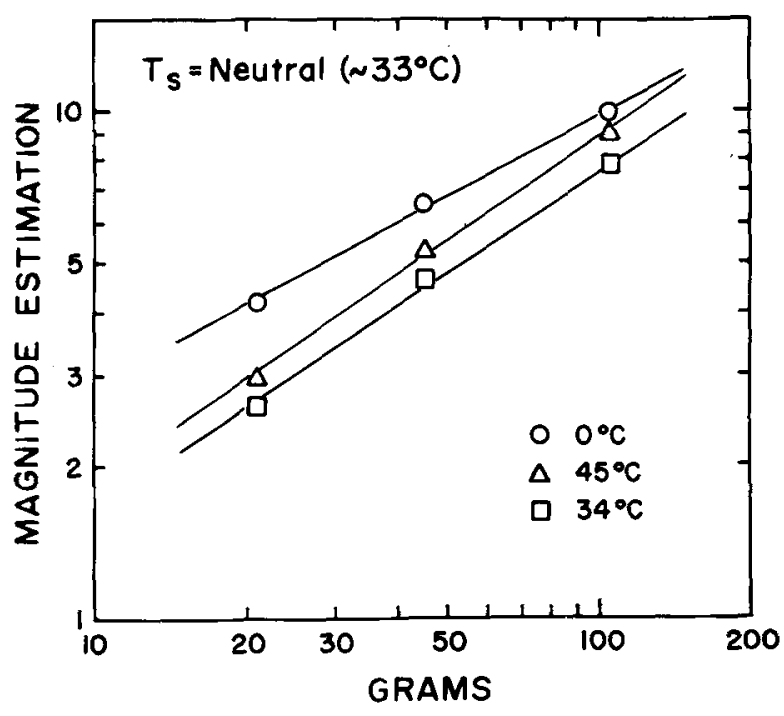

Figure 1. Magnitude estimation of the apparent heaviness of aluminum stimulators placed briefly on the forearm. The parameter is the temperature of the stimulator. The skin was maintained in neutral ambient air conditions (average $33^{\circ} \mathrm{C}$ ). 


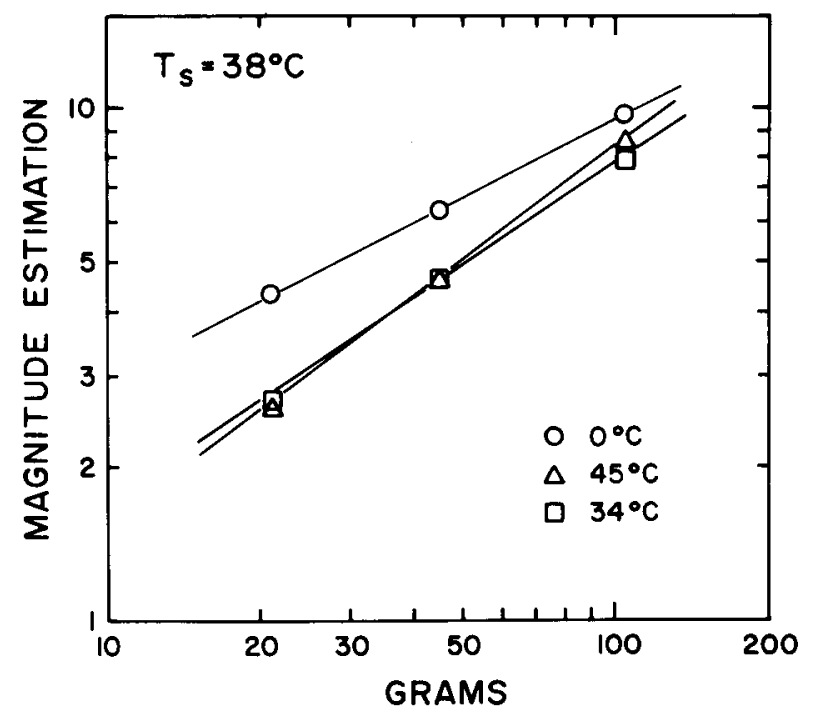

Figure 2. Magnitude estimation of aluminum stimulators placed briefly on neutral-temperature skin $\left(3^{\circ}\right)$ and on skin heated to $38^{\circ} \mathrm{C}$. The parameter is the temperature of the stimulator. Note that the data for the $34^{\circ} \mathrm{C}$ stimulators (squares) are the same as those shown in Figure 1 for neutral skin temperature $\left(33^{\circ}\right)$.

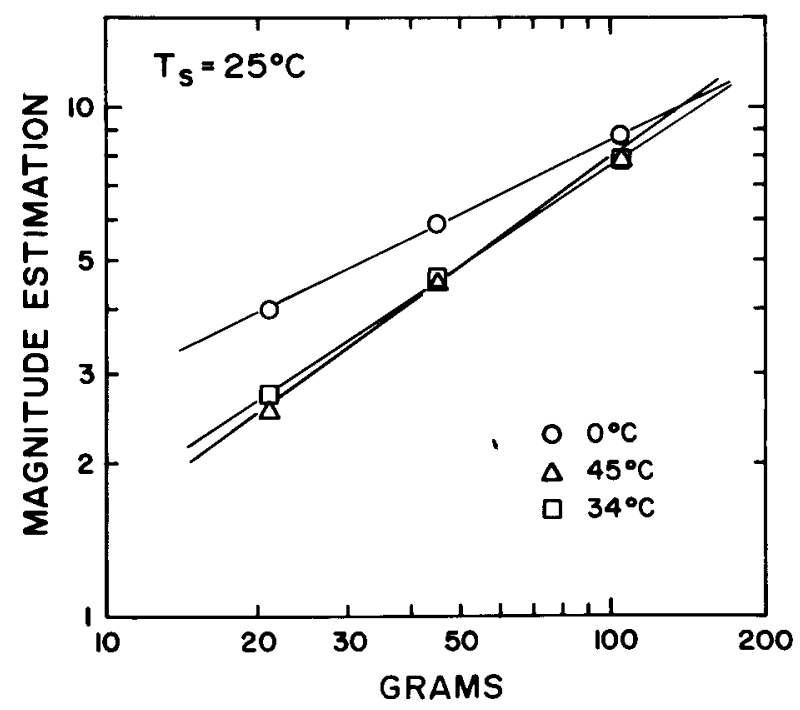

Figure 3. Magnitude estimation of aluminum stimulators placed briefly on neutral-temperature skin $\left(33^{\circ} \mathrm{C}\right)$ and on skin cooled to $25^{\circ} \mathrm{C}$. The parameter is the temperature of the stimulator. Note that the data for the $34^{\circ} \mathrm{C}$ stimulators (squares) are the same as those shown in Figures 1 and 2 for neutral skin temperature $\left(33^{\circ} \mathrm{C}\right)$.

counterpart, at significant levels $(p<.01$ for each of the three pairs).

Figure 2 shows how warming the skin affects the estimated heaviness of the stimulation. It should be pointed out that the control data are the same as in Figure 1. Warming the skin caused little or no difference in the estimation of cold weights - the estima- tions were practically identical to those under neutral conditions and at similar levels of statistical confidence. However, it is clear also that warming the skin essentially eliminated the intensification due to the warmth of objects. This is consistent with an earlier impression (Stevens \& Green, 1978) that systematic heating of the skin by repeated application of the warm stimulators tended to decrease warmth intensification of pressure sensation.

Figure 3 shows how cooling the skin influenced the estimated heaviness of the weights. Cooling reduced intensification produced by both cold and warmth. Intensification produced by warmth was essentially eliminated. Intensification produced by cold, although reduced (by an average of $9.1 \%$ ), was still robust, particularly in the case of the lighter stimulators.

\section{DISCUSSION}

It is quite clear that the perception of heaviness depends upon the combined effects of skin and object temperature. In general, cooling of the skin has an overall effect of reduction of pressure sensation. It is well known, both in everyday experience and in laboratory experiments, that when skin temperature falls substantially below normal there is impairment of tactile functioning. This has been quantified for punctate pressure sensitivity (Stevens, Green, \& Krimsley, 1977), for vibrotactile thresholds (Green, 1977; Weitz, 1941), for tactile spatial acuity (Mackworth, 1953; Mills, 1956; Provins \& Morton, 1960), and for perceived roughness (Green, Lederman, \& Stevens, 1979). Effects of skin warming on the above measures of tactile function (to the extent they have been studied) are small, except that sensitivity to high-frequency vibration peaks a few degrees above normal skin temperature (Green, 1977; Weitz, 1941).

It is not unreasonable that cooling of the skin might also impair perception of pressure. There is reason to believe that the same receptor system that mediates perception of pressure also mediates spatial acuity, a function known to be degraded by cooling of the skin. Stevens (1979a) showed a high correlation between weight sensitivity and two-point limens and error of point localization across body regions. As early as 1834, Weber reported (though no data were provided) that "weights arouse a stronger pressure sensation in those parts of the skin where our tactile acuity for the separation of the compass legs is best"' (Weber, 1978, p. 121).

Despite the dulling effect of the cooling of skin, the sharpening effect of the cooling of objects was large enough to be obvious even when placed on skin considerably below normal temperature. This finding has potential practical significance in the design of tactile aids for the blind. The fact that 
thermal intensification was still able to operate with cold skin suggests that improved thermal sharpening of spatial acuity may also continue to work in the face of a systematic lowering of the skin's temperature that might be brought about by repeated stimulation in a tactile aid. The potential use of cold in this regard looks far more promising than warmth, although a direct measurement of acuity as a function of skin and stimulator temperature would seem to be called for. Heaviness is perceived by warm skin to be about the same as it is by neutral skin. One might argue that hotter stimuli than the ones used might have revealed some intensification. This is possible, but temperatures not much higher than those used here will trigger pain.

The mechanism by which warming of the skin impairs intensification produced by warmth is a matter of speculation, especially since the mechanism of intensification is itself unknown. It seems likely, though, that the difference in temperature between the skin and the weights was simply too small to produce noticeable intensification. This type of explanation would be consistent with the finding that warming the skin had no effect on perception of the cold weights and that warming the skin impairs tactile functioning far less than does cooling it. The observation that the perception of heaviness-and perhaps other tactile features-depends on the temperature of the skin and of the objects contacting the skin suggests that psychophysical and physiological study of the skin senses has often been unrealistically unidimensional. Everyday conditions, however, almost always involve concomitant mechanical and thermal stimulation and a labile skin temperature, especially in the fingers. And, although it is essential to tease out the separate relevant variables, it is also essential to covary them to determine how they act in concert.

Finally, the results of this experiment add fresh evidence to the hypothesis that intensification of the touch sensation by temperature cannot represent a summation of touch sensations and thermal sensations. It is well known that adapting the skin to cold intensifies warmth sensations and adapting the skin to warmth intensifies cold sensations. The sensory summation model would, therefore, predict specifically that cooling the skin ought to increase therinal intensification of warm weights and warming the skin ought to increase thermal intensification of cold weights. Neither of these predictions proved true; in fact, cold adaptation distinctly disrupted thermal intensification of warm weights. This finding fits in with what is known about the way cold adaptation influences touch sensation. That is, the adaptational properties revealed here are typical of the touch modality and inconsistent with those of the thermal modalities.

\section{REFERENCES}

CaIn, W. S. Spatial discrimination of cutaneous warmth. American Journal of Psychology, 1973, 86, 169-181.

Green, B. G. The effect of skin temperature on vibrotactile sensitivity. Perception \& Psychophysics, 1977, 21, 243-248.

Green, B. G., Lederman, S. J., \& Stevens, J. C. The effect of skin temperature on the perception of roughness. Sensory Processes, 1979, 3, 327-333.

MACKWORTH, N. H. Finger numbness in very cold winds. Journal of Applied Physiology, 1953, 5, 533-543.

Mills, A. W. Finger numbness and skin temperature. Journal of Applied Physiology, 1956, 9, 447-450.

Provins, K. A., \& Morton, R. Tactile discrimination and skin temperature. Journal of Applied Physiology, 1960, 15, 155-160.

Stevens, J. C. Thermal intensification of touch sensation: Further extensions of the Weber phenomenon. Sensory Processes, $1979,3,240-248$. (a)

Stevens, J. C. Variation of cold sensitivity over the body surface. Sensory Processes, 1979, 3, 317-326. (b)

Stevens, J. C. Temperature can sharpen tactile acuity. Perception \& Psychophysics, 1982, 31, 577-580.

Stevens, J. C., \& Green, B. G. Temperature-touch interactions: Weber's phenomenon revisited. Sensory Processes, 1978, 2, 206-219.

Stevens, J. C., Green, B. G., \& Krimsley, A. S. Punctate pressure sensitivity: Effects of skin temperature. Sensory Processes, 1977, 1, 238-243.

WEBER, E. H. The sense of touch (English translations of De Tactu and Der Tastsinn by H. E. Ross and D. J. Murray). London: Academic Press, 1978.

WEBE R, E. H. Der Tastsinn und das Gemeingefuhl. In R. Wagner (Ed.), Hnadwörterbuch der Physiologie (Vol. 3). Braunschweig: Vieweg, 1846.

WEITZ, J. Vibratory sensitivity as a function of skin temperature. Journal of Experimental Psychology, 1941, 28, 21-36.

Vendrick, A. J. H., \& Eijkman, E. G. Psychophysical properties which can be related to electrophysiological data. In D. R. Kenshalo (Ed.), The skin senses. Springfield, Ill: Thomas, 1968.

Zimmenmann, R. J., \& STEvens, J. C. Temperature-touch interactions: Is there a reverse Weber phenomenon? Bulletin of the Psychonomic Society, 1982, 19, 269-270.

(Manuscript received March 1, 1982; revision accepted for publication June 23,1982 .) 